\title{
openheart Physiological and prognostic differences between types of exercise stress echocardiography for functional mitral regurgitation
}

\author{
Nobuyuki Kagiyama (D) ,, ${ }^{1,2}$ Misako Toki (D) , ${ }^{3}$ Takuya Yuri, ${ }^{1}$ Shingo Aritaka, ${ }^{3}$ \\ Akihiro Hayashida, ${ }^{1}$ Partho P Sengupta, ${ }^{4}$ Kiyoshi Yoshida ${ }^{1}$
}

- Additional supplemental material is published online only. To view, please visit the journal online (http://dx.doi.org/10. 1136/openhrt-2021-001583).

To cite: Kagiyama N, Toki M, Yuri T, et al. Physiological and prognostic differences between types of exercise stress echocardiography for functional mitral regurgitation. Open Heart 2021;8:e001583. doi:10.1136/ openhrt-2021-001583

NK and MT contributed equally.

NK and MT are joint first authors.

Received 15 January 2021 Revised 3 March 2021 Accepted 12 March 2021

\section{SLinked}

- http://dx.doi.org/10.1136/ openhrt-2021-001641

D Check for updates

(c) Author(s) (or their employer(s)) 2021. Re-use permitted under CC BY. Published by BMJ.

For numbered affiliations see end of article.

Correspondence to Dr Nobuyuki Kagiyama; kgnb_ 27_hot@yahoo.co.jp

\section{ABSTRACT}

Objective Secondary mitral regurgitation (MR) demonstrates dynamic change during exercise. This prospective observational study aimed to compare exercise stress echocardiography (ESE) where handgrip exercise (handgrip-ESE) or semisupine ergometer exercise was performed (ergometer-ESE) for patients with secondary MR.

Methods Handgrip-ESE and symptom-limited ergometerESE were performed for 53 patients (median age (IQR): 68 (58-78) years; 70\% male) on the same day. Baseline global longitudinal strain (GLS) was 9.2\% (6.0\%-14.0\%) and MR volume was $20(14-26) \mathrm{mL}$. All-cause death and cardiac hospitalisation were tracked for median 439 (101-507) days.

Results Handgrip-ESE induced slightly but significantly greater degrees of MR increase (median one grade increase; $p<0.001$ ) than ergometer-ESE, although the changes in other parameters, including GLS $(+1.1 \%$ vs $-0.6 \%, p<0.001$ ), were significantly smaller. Correlations between the two examinations with respect to the changes in the echocardiographic parameters were weak. KaplanMeier analyses revealed poor improvement in GLS during ergometer-ESE, but not the change in MR, was associated with adverse events $(p=0.0065)$. No echocardiographic change observed during handgrip-ESE was prognostic. After adjusting for a clinical risk score, GLS changes during ergometer-ESE remained significant in predicting the adverse events (HR 0.39, $p=0.03$ ) A subgroup analysis in patients with moderate or greater MR at baseline $(n=27)$ showed the same results as in the entire cohort.

Conclusions The physiological and prognostic implications of handgrip-ESE and ergometer-ESE findings significantly differ in patients with left ventricular dysfunction and secondary MR. The type of exercise to be performed in ESE should be carefully selected.

\section{INTRODUCTION}

Secondary mitral regurgitation (MR) is a common complication observed in approximately half of patients with heart failure with left ventricular (LV) dysfunction, ${ }^{1-4}$ and it is

\section{Key questions}

What is already known about this subject?

- Studies have reported that ergometer-exercise stress echocardiography is useful for prognostic assessment in patients with mitral regurgitation. Since ergometer-exercise is often not feasible for elderly and frail patients, doctors sometimes select handgrip exercise as an alternative. However, it has been unknown that handgrip-exercise stress echocardiography is equivalent to ergometer exercise.

What does this study add?

- Our study clearly showed that although handgripexercise stress echocardiography induces similar degree of mitral regurgitation increase, its physiological and prognostic meaning is different from what observed in ergometer-exercise stress echocardiography.

How might this impact on clinical practice?

- Our study raises a caution about handgrip-exercise stress echocardiography as an alternative to ergometer exercise.

associated with a higher incidence of adverse events. ${ }^{5}$ As a recent randomised controlled trial reported that percutaneous intervention for secondary MR may improve the clinical outcomes in appropriately selected populations, the importance of detailed and accurate assessment of secondary MR is being acknowledged. ${ }^{6}$ The dynamic nature of the severity of MR is one of the major difficulties encountered in evaluation of secondary MR. Since the severity of secondary MR is determined by the degree of mitral leaflet tethering caused by papillary muscle dislocation, the degree of secondary MR varies significantly depending on the $\mathrm{LV}$ volume and systolic function, which change often based on the preload and afterload volumes. ${ }^{7-9}$

Exercise stress echocardiography (ESE) for evaluation of valvular heart disease is a 
well-known examination, which allows real-time evaluation of the dynamic changes in the severity of valvular regurgitation and LV function. Previous studies have reported that quantitative assessment of MR and LV function during ergometer exercise is reproducible and has prognostic significance for primary MR. ${ }^{8} 1011$ However, prognostic data of ESE for patients with secondary MR are sparse, although exercise intolerance is a major predictor of adverse events in heart failure. ${ }^{12}{ }^{13}$ In clinical practice, patients with secondary MR, especially those who are indicated for percutaneous mitral valve repair, are often old, frail and intolerant of strenuous exercises. ${ }^{14}$ Hence, handgrip exercise often serves as an alternative in stress tests for such frail patients. ${ }^{15}$ Although the increase in the severity of secondary MR during handgrip exercise is frequently observed in individual cases, ${ }^{15}$ the physiological consequences of handgrip exercise, an isometric exercise, are different from those of common isotonic exercises, like treadmill and ergometer exercises. Thus, this study aimed to investigate (1) the physiological differences between ESE where handgrip exercise was performed (handgrip-ESE) and ESE where semisupine ergometer exercise was performed (ergometer-ESE) in patients with heart failure and secondary MR, and (2) their prognostic implications in such a population.

\section{METHODS}

\section{Study population}

We conducted a prospective single-centre observational study that included patients who underwent ESE in our hospital from October 2015 to December 2016. Consecutive patients with (1) LV ejection fraction (LVEF) $<40 \%$ and secondary MR. Based on the European Society of Cardiology and European Association for Cardiothoracic Surgery guidelines, ${ }^{16}{ }^{17}$ severe MR was defined by comprehensive approach with cut-off values of MR volume $>30 \mathrm{~mL}$ and effective regurgitant orifice $>0.2 \mathrm{~cm}^{2}$; (2) absence of degenerative changes in the mitral leaflet, such as myxomatous change, billowing, prolapse, heavy calcification, infective endocarditis, anomaly and perforation; (3) absence of active ischaemic disease or significant coronary stenosis; and (4) tolerance to at least 25-50 $\mathrm{W}$ of ergometer exercise were enrolled.

All patients underwent laboratory tests for the assessment of the creatinine and B-type natriuretic peptide levels. The Meta-Analysis Global Group In Chronic heart failure (MAGGIC) mortality risk score was calculated, as described previously. ${ }^{18}$ All-cause death and cardiac hospitalisation were tracked via medical chart or telephone interview. The study protocols complied with the guidelines of the Declaration of Helsinki and were approved by the institutional review board. All patients signed the written informed consent forms.

\section{Stress echocardiography}

Baseline echocardiography was performed in the supine position at rest. Handgrip-ESE was subsequently performed in the supine position. The patients were asked to grip a dynamometer with half their maximum strength for $8 \mathrm{~min}$ using either hand, and echocardiographic images were acquired in the last $3 \mathrm{~min}$ of the exercise. The dynamometer indicates a real-time grip strength, and dedicated medical staff was observing the grip strength, confirming that the patient was appropriately keeping the grip strength during the exercise. After the handgrip-ESE, a minimal $30 \mathrm{~min}$ interval was ensured before the patients underwent symptom-limited ergometer-ESE. The workload was initially $25 \mathrm{~W}$ and was then increased by $25 \mathrm{~W}$ every $3 \mathrm{~min}$. Echocardiographic images were acquired at the final workload by an experienced sonographer in the last 1-2 min of the test, as described previously. ${ }^{19}$ During these exercise protocols, the blood pressure and 12-lead ECG were monitored. All echocardiographic examinations were performed using commercially available equipment (Aplio Artida, Canon Medical Systems Corporation, Tochigi, Japan), which was maintained in accordance with the guidelines. ${ }^{20}{ }^{21}$ The LVEF was calculated with the 2D method of disks using the apical 2-chamber and 4-chamber views. The severity of MR was graded using a multiparametric approach, including the ratio of the MR area to the left atrial (LA) area and proximal isovelocity surface area methods, as recommended in the guidelines. ${ }^{22}$ The systolic pulmonary artery pressure (PAP) was calculated by adding $10 \mathrm{~mm} \mathrm{Hg}$ to pressure gradient between the right atrium and right ventricle. ${ }^{10}$ The speckle tracking strain was measured using vendor-independent software (Image Arena, TomTec, Germany), and global longitudinal strain (GLS) was calculated as an average of the 18 segments derived from the three standard apical views. ${ }^{23}$ In the present study, the GLS was expressed in absolute values to avoid confusion, as proposed previously. ${ }^{24}{ }^{25}$ Echocardiographic movies were stored for three heartbeats, and all analyses, including speckle tracking, were performed offline over multiple beats, as required. A trained sonographer or a cardiologist, who was blinded to the patients' clinical information and outcomes, analysed the images according to the guidelines. ${ }^{23}$

\section{Statistical analysis}

The data are presented as the median (IQR) for the continuous variables and as the frequency (\%) for the categorical variables. Group differences were evaluated using the Mann-Whitney $\mathrm{U}$ and $\mathrm{X}^{2}$ or Fisher's exact tests for the continuous and categorical variables, respectively. Differences between the continuous parameters obtained at peak exercise and the baseline values were compared using paired t-tests; the differences noted between these changes during the two tests were also evaluated. The MR grade was compared using the Wilcoxon signedrank test with relevelling of the MR grade to ordinal variables $($ mild $=1$, moderate $=2$, moderate-to-severe $=3$ and severe $=4$ ). The Pearson's correlation tests were used to evaluate the relationship of between the changes in the parameters during handgrip-ESE and those during 
ergometer-ESE. Weighted kappa was used to test the agreement in the MR grade during the two examinations. Moreover, we evaluated the parameters associated with the changes in MR volume and GLS to assess the mechanisms underlying these changes during the two types of exercise using these tests. For survival analysis, the patients were divided according to the median values of the parameters of interest, and the Kaplan-Meier curve analysis, log-rank test and multivariable Cox proportional hazard models were used. As a sensitivity analysis, we repeated all the analyses with a subgroup of patients who had moderate or greater MR.

All statistical analyses were performed with R V.3.5.2 (The R Foundation for Statistical Computing, Vienna, Austria). A two-tailed $\mathrm{p}<0.05$ indicated statistical significance.

\section{Patient and public involvement}

This study was completed without patient involvement.

\section{RESULTS \\ Study cohort}

After excluding one patient due to images of insufficient quality, 106 stress tests were performed for 53 patients whose image quality was sufficient for quantitative analysis. The exercise was standardised and submaximal for all. Table 1 summarises the patient characteristics. The median age was 68 (58-78) years, and $69.8 \%$ of the patients were male, while $45.3 \%$ showed ischaemic aetiology without residual stenosis or active ischaemic episodes. Over one-third of the patients showed significant symptoms of heart failure with New York Heart Association (NYHA) grade III, and the MAGGIC risk score was overall high (30 (23-33)). There was no patient with NYHA IV. At baseline, most patients showed very low LVEF (26\% (16\%-35\%)) and GLS (9.2\% (6.0\%$14.0 \%))$. The severity of secondary MR was mild in $43 \%$, moderate in $42 \%$, and moderate-to-severe or severe in $15 \%$ of the patients. Median MR volume was 20 (14-26) $\mathrm{mL}$ and MR-LA area ratio was $0.12(0.10-0.17)$.

\section{Exercise stress echocardiography}

All patients underwent handgrip-ESE and ergometer-ESE without any complications. None of the patients developed any symptoms of ischaemia, such as chest pain, induced wall motion abnormality or ECG changes. The median workload during ergometer-ESE was $50(50-50)$ $\mathrm{W}$. The significant increase in heart rate $(+40$ and +10 / min by ergometer-ESE and handgrip-ESE, respectively; isotonic exercise does not usually increase heart rate much) and blood pressure $(+38$ and $+25 \mathrm{~mm} \mathrm{Hg}$, respectively) by the exercises indicates that the exercises were properly performed, and the amount of workload was significant. MR volume assessment was feasible for all patients, except for one patient due to insufficient image quality for GLS analysis during ergometer-ESE. The assessment of the tricuspid valve regurgitation peak velocity was not feasible for seven patients due to minimum
Table 1 Patient characteristics

\begin{tabular}{|c|c|}
\hline Parameter & \\
\hline Age, years old & $68(58-78)$ \\
\hline Male, n (\%) & $37(69.8)$ \\
\hline Body mass index $\left(\mathrm{kg} / \mathrm{m}^{2}\right)$ & $23.0(20.1-24.7)$ \\
\hline Systolic blood pressure, mm Hg & $110(99-124)$ \\
\hline Diastolic blood pressure, mm Hg & $69(64-75)$ \\
\hline Heart rate, /min & $69(60-77)$ \\
\hline \multicolumn{2}{|l|}{ Medical history } \\
\hline Ischaemic cardiomyopathy, n (\%) & $24(45.3)$ \\
\hline COPD, n (\%) & $18(34.0)$ \\
\hline Diabetes, n (\%) & $31(58.5)$ \\
\hline \multicolumn{2}{|l|}{ Heart failure severity } \\
\hline NYHA class $\geq 111, \mathrm{n}(\%)$ & $18(34.0)$ \\
\hline B-type natriuretic peptide, $\mathrm{pg} / \mathrm{mL}$ & $284(186-520)$ \\
\hline MAGGIC score & $30(23-33)$ \\
\hline \multicolumn{2}{|l|}{ Echocardiography } \\
\hline Interventricular septum, mm & $10(8-11)$ \\
\hline LV diastolic diameter, mm & $59(52-66)$ \\
\hline LV systolic diameter, mm & $50(42-60)$ \\
\hline Left atrial diameter, mm & $44(39-49)$ \\
\hline LV ejection fraction, \% & $26(16-35)$ \\
\hline Mitral E velocity, $\mathrm{cm} / \mathrm{s}$ & $81(66-102)$ \\
\hline Tissue Doppler e', cm/s & $4.6(3.5-5.7)$ \\
\hline $\mathrm{E} / \mathrm{e}^{\prime}$ & $16.0(12.3-24.6)$ \\
\hline Right atrial pressure, mm Hg & $8(3-8)$ \\
\hline Systolic PAP, mm Hg & $33(29-39)$ \\
\hline GLS, \% (absolute value) & $9.2(6.0-14.0)$ \\
\hline MR volume, $\mathrm{mL}$ & $20(14-26)$ \\
\hline Mitral EROA, $\mathrm{cm}^{2}$ & $0.12(0.10-0.17)$ \\
\hline MR/LA area ratio & $0.21(0.12-0.27)$ \\
\hline Mitral valve tenting height, mm & $9.3(8.0-10.9)$ \\
\hline \multicolumn{2}{|l|}{ MR grade, $\mathrm{n}(\%)$} \\
\hline Mild & $26(49.1)$ \\
\hline Moderate & $19(35.8)$ \\
\hline Moderate-to-severe & $7(13.2)$ \\
\hline Severe & $1(1.9)$ \\
\hline
\end{tabular}

COPD, chronic obstructive pulmonary disease; EROA, effective regurgitant orifice; GLS, global longitudinal strain; LA, left atrium; LV, left ventricular; MAGGIC, Meta-Analysis Global Group In Chronic heart failure; MR, mitral regurgitation; NYHA, New York Heart Association; PAP, pulmonary artery pressure.

regurgitation, and these patients were excluded from analyses using PAP.

Table 2 shows the changes in the vital signs and echocardiographic parameters during handgrip-ESE and ergometer-ESE. As shown in table 2, both the handgrip-ESE and ergometer-ESE showed significant degree of increase in MR, whereas the increase during handgrip-ESE was 
Table 2 Change by each exercise

\begin{tabular}{|c|c|c|c|}
\hline Parameter & Ergometer-ESE & Handgrip-ESE & $\begin{array}{l}\text { P value (ergometer-ESE vs handgrip- } \\
\text { ESE) }\end{array}$ \\
\hline$\Delta$ Heart rate, /min & $+40(+25 \text { to }+52)^{\star *}$ & $+10(5 \text { to } 16)^{\star \star}$ & $<0.001$ \\
\hline$\Delta$ Systolic blood pressure, $\mathrm{mm} \mathrm{Hg}$ & $+38(+22 \text { to }+56)^{\star \star}$ & $+25(16 \text { to } 40)^{\star \star}$ & $<0.001$ \\
\hline$\Delta \mathrm{LV}$ ejection fraction, $\%$ & $+6(+3 \text { to }+9)^{\star \star}$ & $+2(1 \text { to } 4)^{\star \star}$ & $<0.001$ \\
\hline$\Delta$ Stroke volume, $\mathrm{mL}$ & $+11(+4 \text { to }+19)^{\star \star}$ & $-1(-7$ to 3$)$ & $<0.001$ \\
\hline$\Delta \mathrm{E} / \mathrm{e}^{\prime}$ & $+1.0(-1.9$ to 5.3$)$ & $+1.6(-1 \text { to } 3.5)^{\star}$ & 0.26 \\
\hline$\Delta$ Right atrial pressure, $\mathrm{mm} \mathrm{Hg}$ & $+5( \pm 0 \text { to }+5)^{\star \star}$ & $\pm 0( \pm 0 \text { to } \pm 0)^{\star}$ & $<0.001$ \\
\hline$\triangle$ Systolic PAP, mm Hg & $+25(+19 \text { to }+33)^{* \star}$ & $+11(+7 \text { to }+18)^{\star *}$ & $<0.001$ \\
\hline$\Delta \mathrm{GLS}, \%$ (absolute value) & $+1.1(0.3$ to 2.9$) * *$ & $-0.6(-1.3$ to 0.5$)$ & $<0.001$ \\
\hline$\Delta \mathrm{MR}$ volume, $\mathrm{mL}$ & $+9(+1 \text { to }+15)^{\star \star}$ & $+12(+6 \text { to }+16)^{\star *}$ & 0.013 \\
\hline$\Delta \mathrm{MR} / \mathrm{LA}$ area ratio & $+0.07(-0.01 \text { to }+0.13)^{\star *}$ & $+0.06(+0.02 \text { to }+0.10)^{\star \star}$ & 0.59 \\
\hline$\Delta \mathrm{MR}$ grade & $+1(+0 \text { to }+1)^{\star \star}$ & $+1(+1 \text { to }+1)^{\star \star}$ & $<0.001$ \\
\hline
\end{tabular}

${ }^{*} \mathrm{P}<0.05$ vs baseline; ${ }^{*} \mathrm{p}<0.001$ vs baseline. Paired t-tests were used for comparison except for MR grade which was compared using Wilcoxon signed-rank test.

ESE, exercise stress echocardiography; GLS, global longitudinal strain; LA, left atrium; LV, left ventricular; MR, mitral regurgitation; PAP, pulmonary artery pressure.

slightly but significantly greater (MR volume, $+12(6-16)$ $\mathrm{mL}$ vs $+9(1-15) \mathrm{mL}, \mathrm{p}=0.013$, and MR grade $+1(1-1)$ vs $+1(0-1), p<0.001)$. In contrast, the changes in all other parameters, except for $\mathrm{E} / \mathrm{e}^{\prime}(\mathrm{p}=0.26)$, were significantly greater during ergometer-ESE than during handgrip-ESE $(\mathrm{p}<0.001)$. Specifically, the GLS $(-0.6 \%(-1.3 \%$ to $+0.5 \%)$, $\mathrm{p}=0.052 \mathrm{vs}$ baseline $)$ and $\mathrm{LV}$ stroke volume $(-1 \mathrm{~mL} \quad(-7$ to $+3 \mathrm{~mL}$ ), $\mathrm{p}=0.14$ ) did not increase significantly during handgrip-ESE; rather, these values tended to decrease. Figure 1 shows the relationship between the changes in the echocardiographic parameters during handgrip-ESE and ergometer-ESE, and online supplemental figure 1 shows the Bland Altman plots for them. The correlation between the changes during handgrip-ESE and ergometer-ESE was weak or moderate at most $(\mathrm{r}=0.29-0.49)$, and the changes in MR grading during the two examinations showed only moderate degree of agreement (weighted kappa $=0.36$; the same degree of change in $47 \%$, more increase by handgrip in $45 \%$ and more increase by ergometer in $8 \%$ ).

Table 3 summarises the parameters that were significantly correlated with the increase in the GLS and/or MR grade. The parameters associated with the changes in the GLS and/or MR during ergometer-ESE and handgrip-ESE were completely different, suggesting different underlying mechanisms.

\section{Prognostic implication of each examination}

During the median follow-up period of median 439 (101507) days, 28 patients experienced adverse cardiac events, including deaths of seven patients. The Kaplan-Meier curves, with cut-off values of median values as mentioned above, are shown in figure 2. Only poor improvement in the GLS (below median) during ergometer-ESE, but not the change in MR or other parameters, was associated with a higher incidence of adverse events. None of the echocardiographic changes observed during handgrip-ESE had prognostic implications for the patients. Even after adjusting for the MAGGIC risk score, a wellestablished robust risk score for heart failure, a significant increase in the GLS during ergometer-ESE (adjusted HR $0.39,95 \%$ CI 0.16 to $0.91, \mathrm{p}=0.030$ ) was noted (table 4 ).
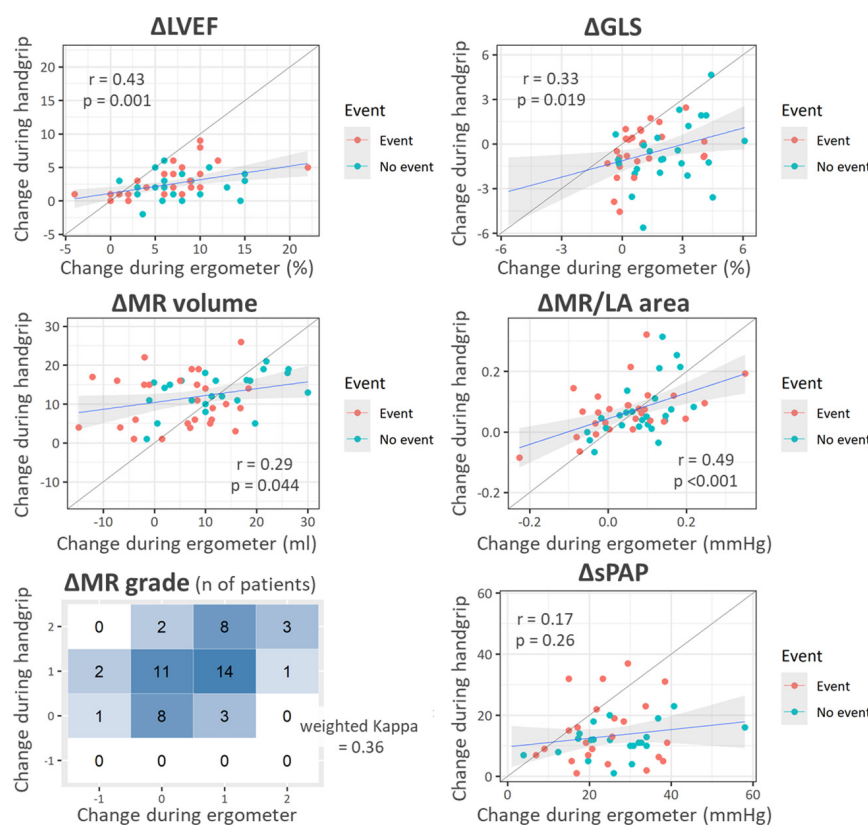

Figure 1 Correlation between the changes during handgripESE and ergometer-ESE. In each panel, the $x$ and $y$ axes show the changes in the parameter during ergometer-ESE and handgrip-ESE. All parameters showed significant but only mild-to-moderate correlation between handgrip-ESE and ergometer-ESE. ESE, exercise stress echocardiography; GLS, global longitudinal strain; LA, left atrium; LVEF, left ventricular ejection fraction; MR, mitral regurgitation; SPAP, systolic pulmonary artery pressure. 
Table 3 Parameters correlated with changes in GLS and MR volume

\begin{tabular}{|c|c|c|c|c|c|c|}
\hline \multirow[b]{2}{*}{ Exercise type } & \multicolumn{3}{|c|}{ Correlation with $\Delta$ GLS } & \multicolumn{3}{|c|}{ Correlation with $\Delta$ MR volume } \\
\hline & Parameter & r $(95 \% \mathrm{Cl})$ & $P$ value & Parameter & r $(95 \% \mathrm{Cl})$ & $P$ value \\
\hline \multirow[t]{8}{*}{ Ergometer-ESE } & $\Delta \mathrm{e}^{\prime}$ & $0.36(0.08$ to 0.58$)$ & 0.012 & $\Delta \mathrm{E} / \mathrm{e}^{\prime}$ & 0.36 (0.09 to 0.58$)$ & 0.010 \\
\hline & $\Delta \mathrm{E}$ & 0.35 ([0.07 to 0.57$)$ & 0.015 & $\Delta \mathrm{E}$ & $0.32(0.04$ to 0.54$)$ & 0.024 \\
\hline & $\triangle \mathrm{sPAP}$ & 0.32 (0.01 to 0.57$)$ & 0.040 & baseline ESV & $-0.28(-0.51$ to -0.01$)$ & 0.040 \\
\hline & baseline sPAP & $-0.34(-0.58$ to -0.04$)$ & 0.027 & baseline E/e' & $-0.35(-0.56$ to -0.08$)$ & 0.011 \\
\hline & baseline $\mathrm{E}$ & $-0.36(-0.58$ to -0.09$)$ & 0.010 & baseline $\mathrm{E}$ & $-0.35(-0.57$ to -0.09$)$ & 0.010 \\
\hline & baseline E/A & $-0.44(-0.68$ to -0.12$)$ & 0.009 & baseline RAP & $-0.38(-0.59$ to -0.12$)$ & 0.006 \\
\hline & & & & baseline $\mathrm{E} / \mathrm{A}$ & $-0.43(-0.66$ to -0.11$)$ & 0.011 \\
\hline & & & & baseline SPAP & $-0.50(-0.69$ to -0.24$)$ & $<0.001$ \\
\hline \multirow[t]{2}{*}{ Handgrip-ESE } & baseline $\mathrm{E} / \mathrm{A}$ & $-0.37(-0.63$ to -0.03$)$ & 0.033 & $\Delta$ diastolic BP & 0.32 (0.05 to 0.55$)$ & 0.021 \\
\hline & $\Delta$ diastolic BP & $-0.44(-0.64$ to -0.19$)$ & 0.001 & & & \\
\hline
\end{tabular}

BP, blood pressure; ESE, exercise stress echocardiography; ESV, end-systolic volume; GLS, global longitudinal strain; MR, mitral regurgitation; RAP, right atrial pressure; SPAP, systolic pulmonary artery pressure.

\section{A subgroup of patients with moderate or greater MR}

Since the degree of baseline MR may impact the results, we performed a sensitivity analysis in patients with moderate or greater MR at baseline $(n=27)$. As summarised in online supplemental table 1 , they were older and had significantly higher B-type natriuretic peptide and MAGGIC risk score, a larger chamber size, higher E/e' and right atrial pressure, more impaired GLS in comparison with those with mild MR. However, the echocardiographic changes during each exercise were similar to the results in the entire cohort (online supplemental table 2) and the correlations between the changes observed during ergometer-ESE and during handgrip-ESE were weak to moderate (online supplemental figure 2). In addition, Kaplan-Meier curve analyses showed the same results that only the lack of improvement in GLS during
ergometer-ESE, but no parameter during handgrip-ESE, was associated with the adverse events (online supplemental figure 3). Univariable Cox analysis also showed the same results as in the entire cohort (online supplemental table 3). Since the number of events (18,67\%) was too small in this subgroup, we did not try multivariable Cox analysis in the subgroup.

\section{DISCUSSION}

This study reported the first direct comparison between two different types of exercise for ESE for patients with heart failure and secondary MR. The results showed that (1) with appropriate amount of exercise indicated by the changes in vital signs, MR was increased slightly but significantly greater during handgrip-ESE than
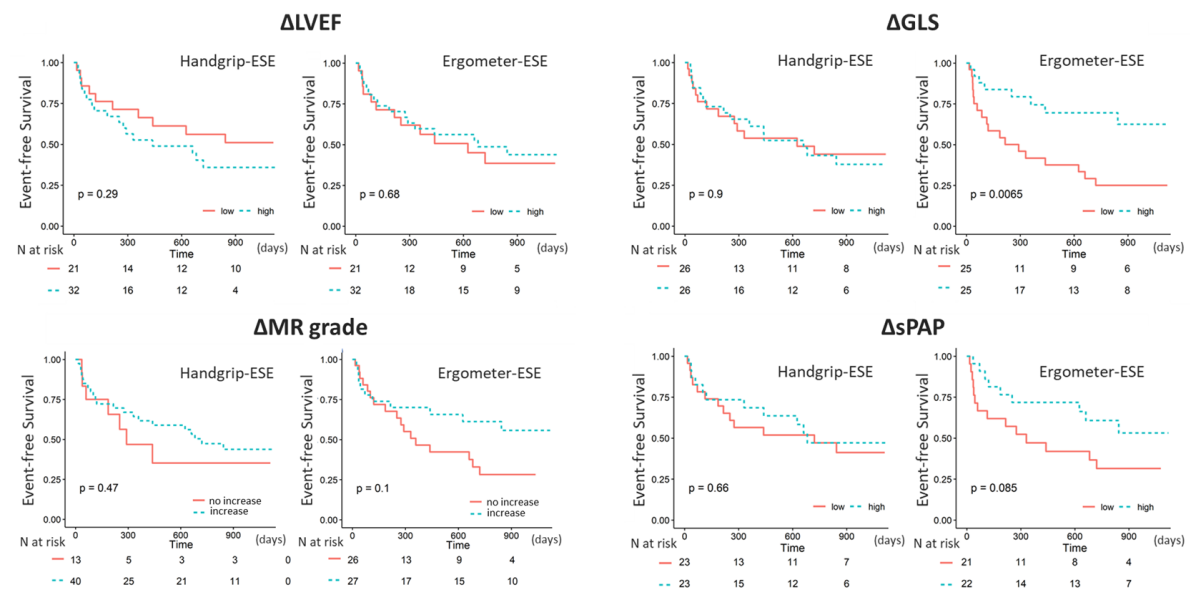

Figure 2 Association of the changes during exercise with clinical outcomes. The Kaplan-Meier curves in each panel show the association of the changes in the parameter during handgrip-ESE (left side) and ergometer-ESE (right side) with all-cause mortality and cardiac hospitalisation. Poor GLS improvement during ergometer-ESE, but not during handgrip-ESE, was significantly associated with a lower adverse event rate. The changes in the LVEF, MR grade and sPAP during either handgripESE or ergometer-ESE did not have prognostic implications for the patients. ESE, exercise stress echocardiography; GLS, global longitudinal strain; LVEF, left ventricular ejection fraction; MR, mitral regurgitation; sPAP, systolic pulmonary artery pressure. 
Table 4 Cox proportional hazard models for adverse events

\begin{tabular}{|c|c|c|c|c|c|}
\hline \multirow[b]{2}{*}{ Exercise type } & \multirow[b]{2}{*}{ Parameter } & \multicolumn{2}{|l|}{ Univariable } & \multicolumn{2}{|c|}{ Adjusted by MAGGIC score } \\
\hline & & HR $(95 \% \mathrm{Cl})$ & $P$ value & HR $(95 \% \mathrm{Cl})$ & $P$ value \\
\hline \multirow[t]{4}{*}{ Ergometer-ESE } & High $\triangle$ LVEF & 0.85 (0.40 to 1.81$)$ & 0.68 & 0.85 (0.40 to 1.80$)$ & 0.67 \\
\hline & High $\Delta$ GLS & 0.33 (0.14 to 0.77$)$ & 0.010 & $0.39(0.16$ to 0.91$)$ & 0.030 \\
\hline & Increased MR grade & $0.52(0.25$ to 1.11$)$ & 0.09 & 0.55 (0.26 to 1.18$)$ & 0.13 \\
\hline & High $\triangle$ sPAP & 0.65 (0.28 to 1.48$)$ & 0.31 & 0.65 (0.29 to 1.49$)$ & 0.31 \\
\hline \multirow[t]{4}{*}{ Handgrip-ESE } & High $\triangle$ LVEF & 1.52 (0.70 to 3.31$)$ & 0.29 & 1.45 (0.67 to 3.17$)$ & 0.35 \\
\hline & High $\Delta \mathrm{GLS}$ & 1.05 (0.50 to 2.20$)$ & 0.90 & 1.04 (0.50 to 2.20$)$ & 0.91 \\
\hline & Increased MR grade & 0.82 (0.33 to 2.04$)$ & 0.68 & 0.60 (0.23 to 1.58$)$ & 0.30 \\
\hline & High $\triangle$ sPAP & 1.03 (0.46 to 2.31$)$ & 0.93 & 1.00 (0.44 to 2.24$)$ & 0.99 \\
\hline
\end{tabular}

Bold type indicates statistical significance $(p<0.05)$.

ESE, exercise stress echocardiography; GLS, global longitudinal strain; LVEF, left ventricular ejection fraction; MAGGIC, Meta-Analysis Global Group In Chronic heart failure; MR, mitral regurgitation; SPAP, systolic pulmonary artery pressure.

ergometer-ESE, while other parameters were changed by less degrees during handgrip-ESE than during ergometer-ESE; (2) the correlation between the changes in the parameters during the two examinations was moderate at most, and factors associated with these changes differed between handgrip-ESE and ergometer-ESE, suggesting different underlying physiological changes during the two examinations; and (3) GLS improvement during ergometer-ESE, but not the change in MR, was associated with better clinical prognosis, while none of the echocardiographic changes observed during handgrip-ESE was prognostic, as summarised in figure 3. These findings were basically the same in the subgroup of the patients with moderate or greater MR at baseline. The study provided novel insights into the understanding of the physiological and prognostic implications of ESE and revealed the differences between the two exercises, thus raising a caution about handgrip-ESE as an alternative to ergometer-ESE.

\section{Physiological changes in cardiac function during exercise}

The dynamic nature of secondary MR in various conditions is well known. Studies have reported that different kinds of stress conditions lead to different alterations in secondary MR:, such that increase in preload and afterload during the acute phase of heart failure commonly increases secondary $M R,{ }^{7}$ whereas pure improvement of cardiac function in response to dobutamine infusion reduces secondary $\mathrm{MR} .{ }^{26}$ Although ergometer exercise is the most well-established exercise for ESE, elderly patients who are candidates for percutaneous valvular intervention are too frail to perform this exercise. Consequently, handgrip-ESE is used as an alternative, but the physiological implications of ergometer-ESE and handgrip-ESE are theoretically very different. While ergometer exercise is isotonic exercise in which large muscles, like the quadriceps and gluteal muscles, are in dynamic motion, resulting in volume overload due to increased venous return, handgrip exercise is isometric exercise

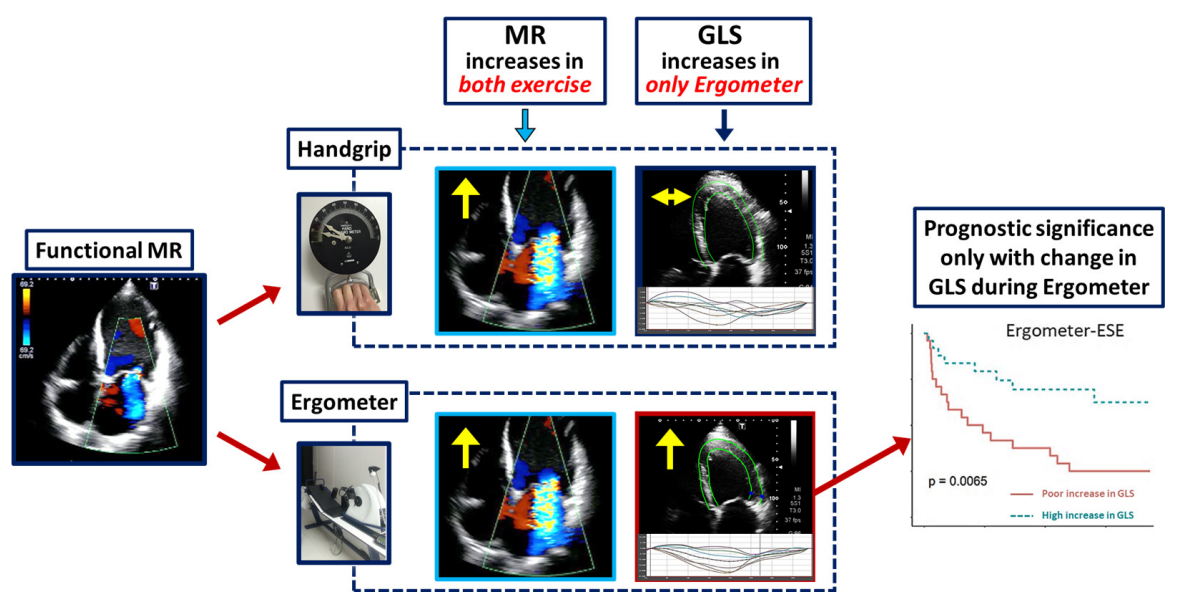

Figure 3 Outcomes of each exercise protocol. Ergometer-ESE resulted in an increase in both the systolic function and MR grade. Poor improvement in GLS was significantly associated with adverse events. Handgrip-ESE resulted in an increase in the MR grade without improvement in the GLS. None of the changes during handgrip-ESE were significantly associated with the patient outcomes. ESE, exercise stress echocardiography; GLS, global longitudinal strain; MR, mitral regurgitation. 
in which the blood pressure and afterload are increased with a lesser increase in the LV preload. ${ }^{27}$ We observed a significant increase in the right atrial pressure and stroke volume during ergometer-ESE, while the increase in the right atrial pressure was minimum and the stroke volume did not change significantly during handgrip-ESE. Our exploratory analysis showed that factors associated with MR and GLS changes during handgrip-ESE were different from those noted during ergometer-ESE. Although various parameters were associated with ergometer-ESE, the main factors in handgrip-ESE were changes in blood pressure. These results suggested that the echocardiographic changes during ergometer-ESE were multifactorial complex outcomes of ergometer exercise, whereas the increase in the blood pressure and afterload was the main trigger for the changes observed during handgrip-ESE. However, since the exploratory analyses were based on speculations without detailed haemodynamic assessment using catheter examinations, further studies are warranted to elucidate the underlying mechanisms.

\section{Changes in the echocardiographic parameters and clinical outcomes}

Although previous studies have reported the prognostic significance of the deterioration in MR, elevated PAP and lack of LV systolic function in primary MR, ${ }^{101128}$ evidence for the significance of ESE in secondary MR is limited and inconsistent. ${ }^{12} 2930$ Ennezat et al reported that the changes in the degree of secondary MR during ergometer exercise did not have prognostic implications for patients with severe $\mathrm{LV}$ dysfunction and mild-to-moderate MR. ${ }^{29}$ Here, we found that change in the GLS during ergometer-ESE, but not during handgrip-ESE, was the only parameter associated with all-cause mortality and cardiac hospitalisation. The changes in MR during either examination were not associated with adverse events.

The conflicting results from the recent COAPT and MITRA-FR trials have prompted an important discussion regarding the candidates appropriate for non-pharmacological intervention for secondary MR. ${ }^{631}$ Therefore, patients with severe MR and relatively preserved LV function might be the best candidates for such interventions, whereas those with mild secondary MR and extremely deteriorated LV might not benefit from such intervention and would not be good therapeutic targets. The results derived from the present population with a relatively milder degree of secondary MR and severe LV dysfunction supported the aforementioned findings. In such patients, the LV function was possibly a more important prognostic determinant than secondary MR. An important implication from our results was that even if MR grade increased during ESE, patients with moderate secondary MR may not be good candidates for non-pharmacological interventions.

\section{Limitations}

Our study has several limitations. First, this was a singlecentre study including a relatively small number of patients. Thus, the present results, especially the part regarding the clinical outcomes, should be considered as a hypothesis-generating pilot study. Further studies are warranted to confirm our findings. Next, treadmill exercise, one of the most popular types of ESE, was not tested in this study. However, since very quick echocardiographic scanning is required in treadmill ESE, it may not pragmatically be a good choice for valvular heart disease assessment. Another concern is that handgrip exercise tends to be insufficient especially in frail patients. However, in the present study, dedicated medical staff was observing the grip strength, confirming that the patient was appropriately keeping the grip strength during the exercise. In addition, the significant increase in MR during handgrip-ESE indicates that the strength of the exercise was substantial. Last, our study population included a substantial number of patients with mild secondary MR. Nevertheless, the subgroup analysis excluding patients with mild MR showed the same results as in the entire cohort. In addition, the importance of ESE only for patients with severe secondary MR at baseline is limited because most such patients are already symptomatic and do not require further stress tests for evaluation.

\section{CONCLUSIONS}

Handgrip-ESE and ergometer-ESE have significantly different physiological and prognostic implications for patients with LV dysfunction and secondary MR. The pattern of cardiac haemodynamic changes during both types of ESE was different, and only improvement in the GLS during ergometer-ESE was associated with adverse events. These results suggest that handgrip-ESE may not be appropriate for risk assessment of patients with secondary MR, although handgrip-ESE is easier to perform. The type of exercise to be performed during ESE should be carefully selected.

\section{Author affiliations}

${ }^{1}$ Department of Cardiology, Sakakibara Heart Institute of Okayama, Okayama, Japan

${ }^{2}$ Department of Cardiovascular Biology and Medicine, Juntendo University, Tokyo, Japan

${ }^{3}$ Department of Clinical Laboratory, Sakakibara Heart Institute of Okayama, Okayama, Japan

${ }^{4}$ Heart and Vascular Institute, West Virginia University, Morgantown, West Virginia, USA

\section{Twitter Nobuyuki Kagiyama @KagiyamaNobu}

Collaborators Hikari Kaneko, Kaoru Sagisawa, Kumiko Ogasawara, Chikako Ikebuchi, Takuya Koyama, Rika Yoneyama, Hiromi Motobe.

Contributors NK—-planning, data acquisition, statistical analysis, writing and all. MT—planning, data acquisition and writing. TY—data acquisition and critical reviewing. SA—data acquisition and critical reviewing. $\mathrm{AH}$-data acquisition and critical reviewing. PPS—writing and critical reviewing. KY—planning, writing and critical reviewing.

Funding This work was partially supported by JSPS KAKENHI Grant-in-Aid for Research Activity Start-up (grant number: 20K22504).

Competing interests NK is affiliated with and receives salary from a department funded by Philips Healthcare; Asahi KASEl Corporation; Inter Reha Co and Toho 
Holdings Co based on collaborative research agreements. PPS is a consultant to HeartSciences, Ultromics and Kencor Health.

Patient consent for publication Not required.

Ethics approval The institutional review board of the Sakakibara Heart Institute in Okayama.

Provenance and peer review Not commissioned; externally peer reviewed.

Data availability statement Data are available upon reasonable request directly to the authors.

Open access This is an open access article distributed in accordance with the Creative Commons Attribution 4.0 Unported (CC BY 4.0) license, which permits others to copy, redistribute, remix, transform and build upon this work for any purpose, provided the original work is properly cited, a link to the licence is given, and indication of whether changes were made. See: https://creativecommons.org/ licenses/by/4.0/.

\section{ORCID iDs}

Nobuyuki Kagiyama http://orcid.org/0000-0002-2305-0049

Misako Toki http://orcid.org/0000-0003-2915-660X

\section{REFERENCES}

1 Feinberg MS, Schwammenthal E, Shlizerman L, et al. Prognostic significance of mild mitral regurgitation by color Doppler echocardiography in acute myocardial infarction. Am J Cardiol 2000;86:903-7.

2 Nappi F, Avatar Singh SS, Santana O, et al. Functional mitral regurgitation: an overview for surgical management framework. $J$ Thorac Dis 2018;10:4540-55.

3 Magne J, Sénéchal M, Dumesnil JG, et al. Ischemic mitral regurgitation: a complex multifaceted disease. Cardiology 2009;112:244-59.

4 Bursi F, Enriquez-Sarano M, Nkomo VT, et al. Heart failure and death after myocardial infarction in the community: the emerging role of mitral regurgitation. Circulation 2005;111:295-301.

5 Grigioni F, Enriquez-Sarano M, Zehr KJ, et al. Ischemic mitral regurgitation: long-term outcome and prognostic implications with quantitative Doppler assessment. Circulation 2001;103:1759-64.

6 Stone GW, Lindenfeld J, Abraham WT, et al. Transcatheter mitral-valve repair in patients with heart failure. $N$ Engl J Med 2018;379:2307-18.

7 Wada Y, Ohara T, Funada A, et al. Prognostic impact of functional mitral regurgitation in patients admitted with acute decompensated heart failure. Circ J 2016;80:139-47.

8 Lebrun F, Lancellotti P, Piérard LA. Quantitation of functional mitral regurgitation during bicycle exercise in patients with heart failure. $J$ Am Coll Cardiol 2001;38:1685-92.

9 Kagiyama N, Shrestha S. Echocardiographic assessment of mitral regurgitation. J Med Ultrason 2020;47:59-70.

10 Magne J, Lancellotti P, Piérard LA. Exercise pulmonary hypertension in asymptomatic degenerative mitral regurgitation. Circulation 2010;122:33-41.

11 Magne J, Mahjoub H, Dulgheru R, et al. Left ventricular contractile reserve in asymptomatic primary mitral regurgitation. Eur Heart $J$ 2014;35:1608-16.

12 Garbi M, Chambers J, Vannan MA, et al. Valve stress echocardiography: a practical guide for referral, procedure, reporting, and clinical implementation of results from the HAVEC group. JACC Cardiovasc Imaging 2015;8:724-36.

13 Del Buono MG, Arena R, Borlaug BA, et al. Exercise intolerance in patients with heart failure: JACC state-of-the-art review. J Am Coll Cardiol 2019;73:2209-25.

14 Arnold SV. Frail Elderly, the Ideal Patients for MitraClip. JACC Cardiovasc Interv 2017;10:1930-1.
15 Higuchi R, Mahara K, Saji M, et al. Severe functional mitral regurgitation manifested by isometric handgrip: revival of simple and non-invasive stress test in the era of transcatheter mitral valve repair Cardiovasc Interv Ther 2020;35:417-8.

16 Joint Task Force on the Management of Valvular Heart Disease of the European Society of Cardiology (ESC), European Association for Cardio-Thoracic Surgery (EACTS), Vahanian A, et al. Guidelines on the management of valvular heart disease (version 2012). Eur Heart $J$ 2012;33:2451-96.

17 Baumgartner H, Falk V, Bax JJ, et al. 2017 ESC/EACTS guidelines for the management of valvular heart disease. Eur Heart $J$ 2017;38:2739-91.

18 Pocock SJ, Ariti CA, McMurray JJV, et al. Predicting survival in heart failure: a risk score based on 39372 patients from 30 studies. Eur Heart J 2013;34:1404-13.

19 Lancellotti P, Pellikka PA, Budts W, et al. The clinical use of stress echocardiography in non-ischaemic heart disease: recommendations from the European association of cardiovascular imaging and the American Society of echocardiography. Eur Heart $J$ Cardiovasc Imaging 2016;17:1191-229.

20 Nakatani S, Akaishi M, Asanuma T, et al. Guidelines from the Japanese Society of echocardiography: guidance for the management and maintenance of echocardiography equipment. $J$ Echocardiogr 2015;13:1-5.

21 Daimon M, Akaishi M, Asanuma T, et al. Guideline from Japanese Society of echocardiography: 2018 focused update incorporated into guidance for the management and maintenance of echocardiography equipment. J Echocardiogr 2018;16:1-5.

22 Zoghbi WA, Adams D, Bonow RO, et al. Recommendations for noninvasive evaluation of native valvular regurgitation: a report from the American Society of echocardiography developed in collaboration with the Society for cardiovascular magnetic resonance. J Am Soc Echocardiogr 2017;30:303-71.

23 Lang RM, Badano LP, Mor-Avi V, et al. Recommendations for cardiac chamber quantification by echocardiography in adults: an update from the American Society of echocardiography and the European association of cardiovascular imaging. Eur Heart J Cardiovasc Imaging 2015;16:233-71. e14.

24 Kagiyama N, Sugahara M, Crago EA, et al. Neurocardiac injury assessed by strain imaging is associated with in-hospital mortality in patients with subarachnoid hemorrhage. JACC Cardiovasc Imaging 2020;13:535-46.

25 Flachskampf FA, Blankstein R, Grayburn PA, et al. Global Longitudinal Shortening: A Positive Step Towards Reducing Confusion Surrounding Global Longitudinal Strain. JACC Cardiovasc Imaging 2019;12:1566-7.

26 Keren G, Laniado S, Sonnenblick EH, et al. Dynamics of functional mitral regurgitation during dobutamine therapy in patients with severe congestive heart failure: a Doppler echocardiographic study. Am Heart J 1989;118:748-54.

27 Bond V, Curry BH, Adams RG, et al. Cardiovascular responses to an isometric handgrip exercise in females with prehypertension. $N A m ~ J$ Med Sci 2016;8:243-9.

28 Suzuki K, Izumo M, Yoneyama K, et al. Influence of exercise-induced pulmonary hypertension on exercise capacity in asymptomatic degenerative mitral regurgitation. J Cardiol 2015;66:246-52.

29 Ennezat PV, Maréchaux S, Huerre C, et al. Exercise does not enhance the prognostic value of Doppler echocardiography in patients with left ventricular systolic dysfunction and functional mitral regurgitation at rest. Am Heart J 2008;155:752-7.

30 Lancellotti P, Troisfontaines P, Toussaint A-C, et al. Prognostic importance of exercise-induced changes in mitral regurgitation in patients with chronic ischemic left ventricular dysfunction. Circulation 2003;108:1713-7.

31 Pibarot P, Delgado V, Bax JJ. MITRA-FR vs. COAPT: lessons from two trials with diametrically opposed results. Eur Heart J Cardiovasc Imaging 2019;20:620-4. 